\title{
Photochemical synthesis and optical properties of high membered thiohelicenes
}

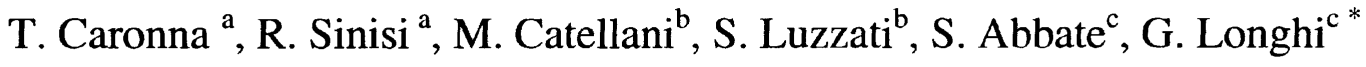 \\ a Dipartimento di Chimica del Politecnico, Via Mancinelli 7, 20131 Milano, Italy \\ ${ }^{\mathrm{b}}$ Istituto di Chimica delle Macromolecole-CNR. Via Bassini 15, 20133 Milano, Ital y \\ ${ }^{c}$ Dip. Scienze Biomediche e Biotecnologie, Università di Brescia, Via Valsabbina 19, 25124 Brescia, Italy
}

\begin{abstract}
A general photochemical synthesis of large thiohelicenes composed containing 5, 7, 9 and 11 rings is presented. The absorption and emission properties of the racemic molecules are discussed along with the chirooptical properties of two enantiomers.
\end{abstract}

Keywords: Thiohelicenes, Photochemistry, Synthesis, Absorption, Emission, Circular Dicroism

\section{Introduction}

Helicenes are intrinsically chiral molecule formed by ortho- fused aromatic rings. Their polyconjugated helical structure makes them interesting candidates for optoelectronic applications [1] and for molecular devices [2]. Nonracemic helicenes exhibit an organisation in macroscopic fibrous structures in the solid state, while in concentrated solutions and thin films a gigantic rotation of the plane of polarised light was evidenced [3].

In this communication we report on a general synthetic approach we used to prepare a series of heterohelicenes formed by alternate thiophene and benzene rings [4]: trithia[5]heterohelicene $\mathbf{A}$, tetrathia[7]heterohelicene $\mathbf{B}$ and, for the first time, large molecules such as pentathia [9]heterohelicene $\mathbf{C}$ and esathia[11] heterohelicene D. We have separated the enantiomers of $\mathrm{C}$ and their chirooptical characterisation is reported along with the absorption and emission spectra of racemic mixture

\section{Experimental}

The thiohelicenes preparation is a laborious process that requires a photochemical condensation as crucial step in which the very dilute conditions limit a large scale preparation. Two approaches were possible to synthesise large condensed systems. The first consists in the double photochemical ring closure of a bis(1,2-diheteroaryl) ethylene, the second in a single photochemical cyclisation of a 1,2-diheteroaryl-ethylene. We preferred the latter approach, in order to maximise the yield and increase the rate in the photochemical step. In scheme 1 is reported the synthesis of the thiohelicene series.
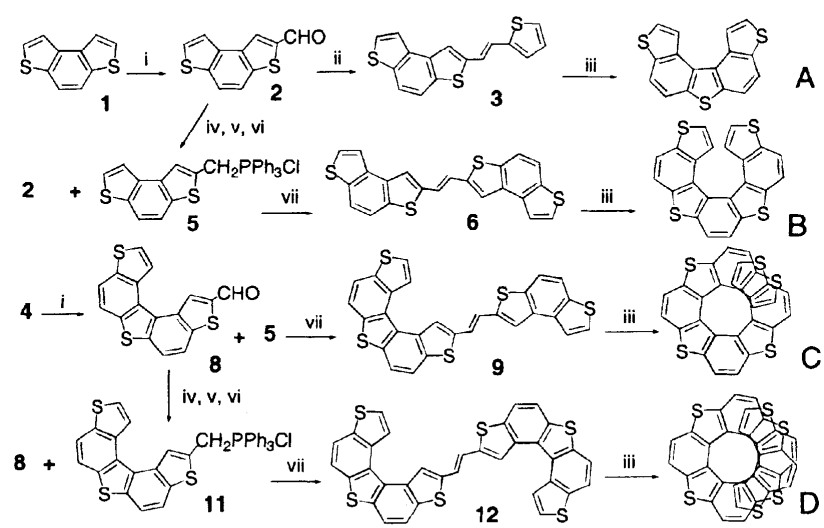

Scheme 1. (i) $\mathrm{POCl}_{3}, \mathrm{PhN}(\mathrm{Me}) \mathrm{CHO}$, toluene; (ii) $\left(\mathrm{C}_{4} \mathrm{H}_{3} \mathrm{~S}\right) \mathrm{CH}_{2} \mathrm{PPh}_{3} \mathrm{Br}$, $\mathrm{NaOMe}$, anhydrous $\mathrm{MeOH}$; (iii) hv $350 \mathrm{~nm}$ or visible light; (iv) $\mathrm{NaBH}_{4}$, $\mathrm{EtOH} / \mathrm{THF}$; (v) $\mathrm{SOCl}_{2}$, benzene/Py; (vi) $\mathrm{PPh}_{3}$, benzene; (vii) $\mathrm{NaOMe}$, anhydrous $\mathrm{MeOH}$.

* Corresponding author. Tel: +39-030-3717-411; fax: +39-030-3701-157; E-mail: longhi@med.unibs.it 
The synthesis of thio[n]helicene $(\mathrm{n}=7,9$ condensed rings) starts by reacting thio[n-4] helicene with Vilsmeier reagent ( $\mathrm{POCl}_{3}$ and $\mathrm{PhN}(\mathrm{Me}) \mathrm{CHO}$ in boiling toluene) in order to obtain the corresponding 2-carboxyaldehyde. The phosphonium salt 2 is produced from the 2carboxyaldehyde 1 with a sequence of reactions illustrated in Scheme 1. The Wittig reaction between the phosphonium salt and the 2-carboxyaldehyde of the thio[n4] helicene gives a 1,2-diheteroarylethylene. The racemic thio[n]helicene can be easily obtained with high yield by the photoinduced cyclodehydrogenation. The synthesis of the larger esathia[11] helicene $\mathbf{D}$ begins with the reaction between the 2-carboxyaldehyde-thio[5]helicene 3 and the phosphonium salt of the thio[5]helicene, followed by photocyclisation of the 1,2-diheteroarylethylenes.

\section{Optical properties}

Electronic absorption spectra of the thiohelicene series in solution are reported in figure 1. Increasing the number of condensed rings, the absorption bands shift to lower energy due to the lengthening of the $\pi$-conjugation in the helical system. The photoluminescence spectra shown in figure 2 exhibit a similar behaviour, with emissions that are red-shifted increasing the molecular size.

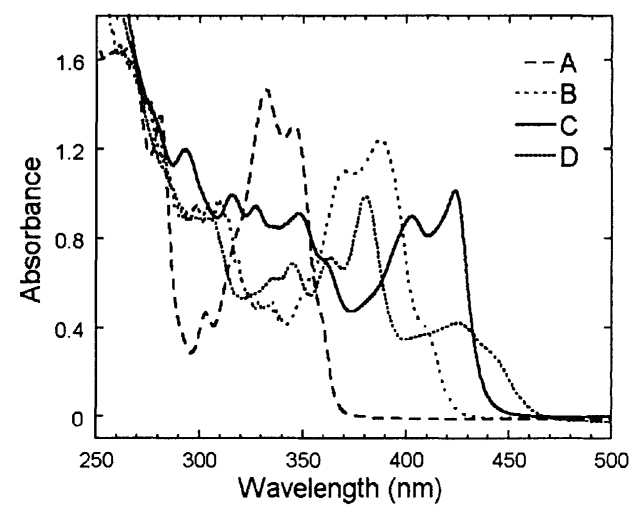

Fig. 1. Electronic absorption spectra in $\mathrm{CHCl}_{3}$ solution

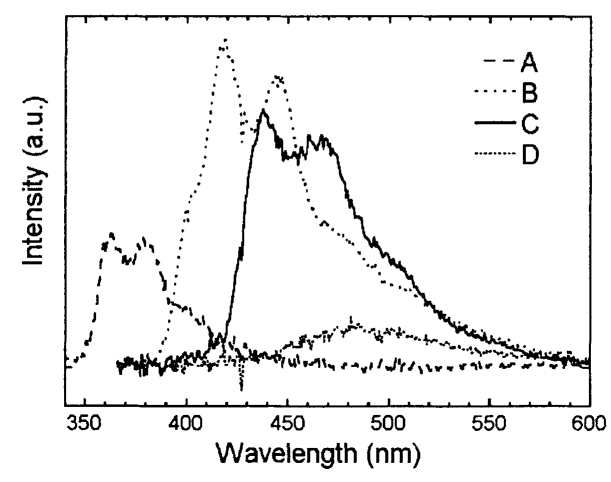

Fig. 2. Emission spectra in $\mathrm{CHCl}_{3}$ solution

\section{Chirooptical properties}

The enantiomers of pentathia[9]heterohelicene $\mathbf{C}$ were separated by Hplc cromatography using a CHIRACEL OD analytical column with hexane/toluene $4 / 1$ as eluent.

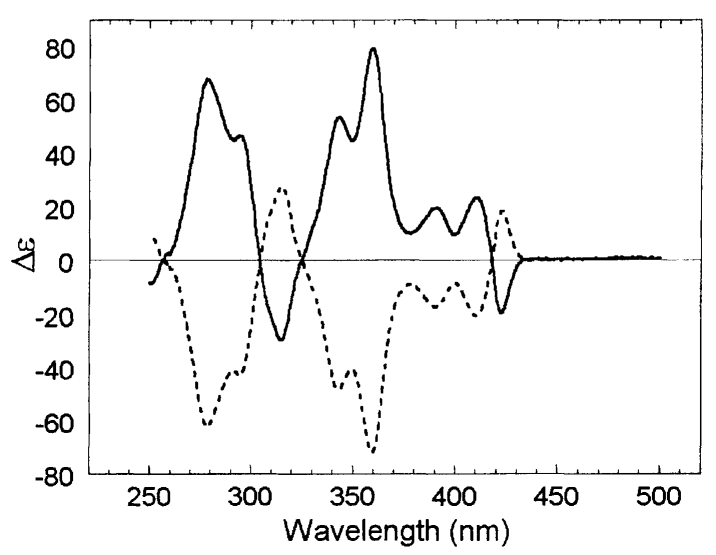

Fig. 3. $\mathrm{CD}$ of the two enantiomers of thiohelicene $\mathbf{C}$ in $\mathrm{CHCl}_{3}$ solution. (+)-C $2.5 \times 10^{-5} \mathrm{M}$, (-)-C $3 . \times 10^{-5} \mathrm{M}$

We report in figure 3 the $C D$ spectra of the two enantiomers of thiohelicene $\mathbf{C}$ in $\mathrm{CHCl}_{3}$ solutions obtained on a Jasco J500 spectrometer. Comparing with the results of references $[5,6]$ we assign the $\mathrm{CD}$ spectra of the $(+)$ and $(-)$-enantiomer. It is commonly accepted [5, 7], from theoretical and experimental evidence, that $(+)$-helicenes and $(+)$-heterohelicenes correspond to the absolute configuration of a right-handed helix ( $\mathrm{P}$ configuration).

If one assumes the spectroscopic labelling adopted in the literature [5], one can recognize in both absorption and CD spectra the typical $\alpha, p, \beta, \beta$ ' bands of condensed aromatic hydrocarbons. The $\alpha$ band of $(+)-C$, which is hidden by the $\mathrm{p}$ bands in the absorption spectrum, gives negative $\mathrm{CD}$ at $423 \mathrm{~nm}$. The $\mathrm{p}$ bands $(410,391 \mathrm{~nm})$, corresponding to the HOMO-LUMO transition, are very intense in the absorption spectrum, and give weak positive $\mathrm{CD}$. Finally, two strong structured positive $\mathrm{CD}$ bands $\beta$ (359 and 343 $\mathrm{nm})$ and $\beta^{\prime}(295$ and $278 \mathrm{~nm})$ are present.

\section{References}

[1] D. Beljonne, Z. Shuai, J.L. Brédas, M Kauranen, T Verbiest, A Persoons, J. Chem Phys. 108 (1998) 1301

[2] T.R. Kelly, J. P. Sestelo, I. Tellitu, J. Org. Chem., 63 (1998) 3655

[3] a) C. Nuckolls, T. J. Katz and L. Castellanos, J.Am. Chem. Soc., 118 (1996) 3767; b) C. Nuckolls, T. J. Katz, T. Verbiest, S.Van Elshocht, H-G. Kuball, S. Kiesewalter, A. J. Lovinger, A.Persoons, J. Am. Chem. Soc., 120 (1998) 8656

[4] T. Caronna, R. Sinisi, M. Catellani, L. Malpezzi, S. V. Meille, A Mele, J. Chem. Soc.,Chem Commun, (2000) 1139

[5] M.B.Groen,H.Wynberg , J.Am.Chem.Soc., 93 (1971) 2968

[6] K.Tanaka, H.Suzuki, H.Osuga, J.Org.Chem., 62 (1997) 4465

[7] D.A.Lightner,D.T.Hefelfinger,T.WPowers, G.W.Frank,K.N.Trueblood, J.Am.Chem.Soc., 94 (1972) 3492 